\section{Visual outcome following cataract extraction in patients aged 90 years and older}

\begin{abstract}
Purpose To analyse surgical outcomes following cataract surgery in patients aged 90 years and older.

Methods A retrospective case series of 78 patients aged 90 years or more (82 eyes) undergoing cataract extraction between 2000 and 2006 was performed. Age, sex, ocular comorbidities, surgical reports, preoperative and postoperative visual acuity (VA), and postoperative complications were recorded. A comparison of visual outcome between different ocular comorbidity groups was performed.
\end{abstract}

Results Average age was 91.8 years. The most common comorbidities were dry AMD (47.4\%) and open-angle glaucoma $(24.4 \%)$. No ocular comorbidity was found in only 22 patients (28.2\%). Posterior capsular tear $\mathbf{( 8 . 5 \% )}$ was the most prevalent operative complication.

Department of

Ophthalmology, Meir Medical Center, Kfar Saba, Israel

Correspondence: E Rosen, Department of

Ophthalmology, Meir Medical Center, 59 Tshernichovsky st., Kfar-Saba 44281, Israel

Tel: + 97297472154 ; Fax: + 97297472427

E-mail: ermd14@

gmail.com

Received: 3 March 2008 Accepted in revised form: 1 June 2008

Published online: 4 July 2008

The authors have no proprietary interest
Overall VA improvement was $67.8 \%$, whereas unchanged VA and VA worsening rates were $16.1 \%$ each. A total mean improvement of $0.63 \log$ MAR was achieved (from preoperative $1.36 \log$ MAR to postoperative $0.73 \log$ MAR), whereas patients with no comorbidity achieved as high as $0.8 \log$ MAR improvement (1.42-0.62 logMAR). Almost $17 \%$ of patients achieved uncorrected VA of 20/40 or better at day 7, compared to $7 \%$ preoperatively. Final VA of 20/40 or better was achieved in $25 \%$ of the patients. AMD patients showed lower final improvement rates and higher rates of unchanged VA, as compared to the no comorbidity and glaucoma group (statistically significant at day 7).

Conclusions Overall, approximately $70 \%$ of very elderly patients can achieve VA improvement, which rises to $82 \%$ in those without ocular comorbidity. Although patients with AMD show less improvement and more
E Rosen, A Rubowitz and El Assia

unchanged VA outcome rates, $62.5 \%$ can still enjoy improvement in VA.

Eye (2009) 23, 1120-1124; doi:10.1038/eye.2008.203; published online 4 July 2008

Keywords: cataract extraction; visual outcome; very elderly patients

\section{Introduction}

As the average lifespan and number of both the elderly and the very elderly population continues to increase in most of the western world, the number of very elderly patients undergoing cataract surgery will undoubtedly increase as well. This trend raises several questions: Is there an age limit for cataract surgery? Is there a different profile of intraoperative or postoperative complications in this subpopulation, and how does their surgical outcome differ from that of younger patients?

The relatively few studies regarding these questions show varying and often conflicting findings, and the small sample sizes of some of them, in addition to the heterogeneous sample population in several other health insurance or national registry-based cohorts, have made comparisons difficult. We herein report a study of 82 consecutive cataract surgeries performed on patients aged 90 years and older, over a period of 6 years in our ophthalmology department, all using similar surgical techniques and preoperative and postoperative care and monitoring.

\section{Materials and methods}

A consecutive series of all patients aged at least 90 years old at the time of surgery, undergoing cataract surgery at our Eye Department from January 2000 to March 2006 was studied. Data extracted from patient files included age, sex, 
ocular comorbidity, surgical reports (with special emphasis on intraoperative complications and difficulties), preoperative and postoperative visual acuity (VA), and the need for additional surgeries or postoperative treatments for complications. As our institution is a regional referral center, most patients are seen 1 day and 1 week postoperatively. Patients without major ocular comorbidity and with a normal postoperative course are released from our service to continue follow-up and care with their community-based ophthalmologists. Patients with complications requiring further care or those with coexisting ocular morbidity requiring tertiary care and follow-up are seen as needed for varying lengths of time as their medical condition dictates. Available data included preoperative VA and 1 week postoperatively in all cases. For those patients remaining in our care, later follow-up and VAs were available. Data of patients released from our care 1 week after surgery were retrieved when possible from their community ophthalmologists.

Statistical analysis was performed using the analysis of variance (ANOVA), Pearson $\chi^{2}$ test and $t$-test. Snellen VAs were converted into a logMAR for analysis. $P<0.05$ was considered statistically significant.

\section{Results}

In this retrospective study, the data of 78 patients who had 82 cataract surgeries were collected. Their average age was 91.8 years (range: 90-103, median-91). An unexpected finding was male predominance $(52.6 \%$, $41 / 78)$, considering the longer life expectancy of women. Four patients had two operations, one in each eye.

Ocular comorbidity details were available in all cases. The most common pathologies were: age-related macular degeneration (AMD) - 37 patients, open-angle glaucoma - 19 patients, and pseudoexfoliation (PXF) syndrome - 12 patients. No ocular comorbidity was found in only 22 patients. Table 1 summarizes the ocular comorbidity data.

Operative parameters included the anaesthesia type (89\% - topical, 8.6\% - local, and the rest in general anaesthesia), surgery type $(91.5 \%$ - phacoemulsification, $8.5 \%$ - manual extracapsular cataract extraction), and implanted lens type $(91.5 \%$ - posterior chamber, $8.5 \%$ - anterior chamber). In all surgeries, the planned target refraction was between $-0.5 \mathrm{D}$ to $-1 \mathrm{D}$.

Operative complications included posterior capsular tear in 7 of 82 operations $(8.5 \%)$, severe phacodonesis and zonulolysis in three patients (3.7\%), and anterior capsular tear, choroidal detachment, dropped lens particle, and counterclockwise IOL insertion (one of each).

Preoperative pinhole VA was compared to the VA at day 7 and at final follow-up. Overall, the VA
Table 1 Preoperative ocular comorbidity data (78 patients)

\begin{tabular}{llc}
\hline Comorbidity & & $(\%)$ \\
\hline No ocular comorbidity & & $22(28.2)$ \\
AMD & Dry & $37(47.4)$ \\
& Wet & $1(1.3)$ \\
& Disciform scar & $1(1.3)$ \\
Open-angle glaucoma & Primary & $16(20.5)$ \\
& PXF & $3(3.8)$ \\
Diabetic retinopathy & Non proliferative & $3(3.8)$ \\
Angle-closure glaucoma & Phacomorphic & $1(1.3)$ \\
PXF syndrome and zonulolysis & & $12(15.4)$ \\
Trachoma & & $1(1.3)$ \\
Other potential vision affecting & Total & $11(14.1)$ \\
pathologies & & \\
& Corneal opacity & $2(2.6)$ \\
& Amblyopia & $2(2.6)$ \\
& High myopia & $2(2.6)$ \\
& Retinal & $1(1.3)$ \\
& detachment & \\
& Epiretinal & $1(1.3)$ \\
& membrane & \\
& Corneal oedema & $1(1.3)$ \\
& Optic atrophy & $1(1.3)$ \\
& Asteroid hyalosis & $1(1.3)$ \\
\hline & &
\end{tabular}

improvement rate at day 7 was $59.2 \%$. The most frequent VA at day 7 was 20/200. VA of 20/40 or better was recorded in $7.0 \%$ before surgery and in $16.9 \% 7$ days after surgery.

Longer follow-up results were available for 56 surgeries: 40 from patients remaining in our care, and 16 from community health-care providers. The mean follow-up time was $6.75 \pm 7.65$ months and the median follow-up time was 2 months. Overall, $67.8 \%$ showed an improvement, $16.1 \%$ a worsening and $16.1 \%$ an unchanged VA (as compared to the preoperative data), whereas higher improvement rate $(75 \%)$ and lower worsening rate $(6.3 \%)$ were found in the community health-care patients' data. Figures 1 and 2 summarize VA data. Mean preoperative and postoperative VA were $1.36 \log$ MAR ( 20/460) and 0.73 logMAR ( 20/108), respectively. The comorbidity impact on VA outcome is detailed in Table 2. AMD patients showed lower improvement rates and higher unchanged VA rates, as compared with patients with open-angle glaucoma or without ocular comorbidity (statistically significant at day 7). Nevertheless, AMD patients achieved an average improvement of $0.49 \log \mathrm{MAR}$, as compared to 0.80 in patients without comorbidity (VA data are detailed in Table 3). The difference was not statistically significant.

Early (less than 7 days) postoperative complications included high IOP, anterior chamber fibrin, wound leakage, and haptic subluxation into the anterior chamber in one case each (1.3\% of 77). Macular oedema and high astigmatism (1 case each) were found as late complications. 


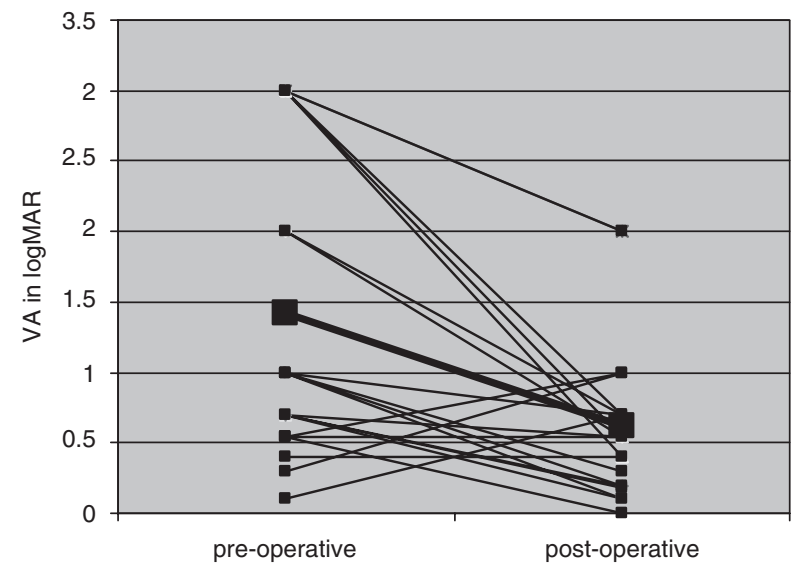

Figure 1 Changes in visual acuities (VAs; logMAR) from preoperation to final follow-up for patients without any comorbidity. Most of the patients show a certain improvement in VA. The mean change is emphasized.

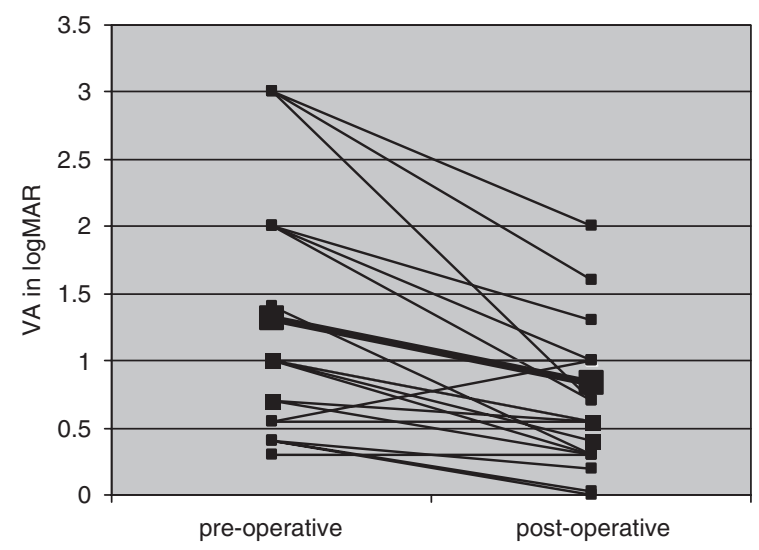

Figure 2 Changes in visual acuities (logMAR) from preoperation to final follow-up for AMD patients. The improvement is certain as well, although the mean change is lower than in Figure 1 (statistically insignificant).

\section{Discussion}

Several previous studies have studied the issue of cataract surgery outcomes in very elderly patients. Lundstrom $^{1}$ et al studied the outcomes of 757 cataract surgery patients over 85 years old, extracted from a national cataract surgery registry. Owing to the nature of national cohort-based data, patients were examined and treated in many different clinics, with varying proportions of surgical techniques utilized and high rates of incomplete follow-up. This study concluded that postoperative VA was improved in some $84 \%$ of eyes operated on, and to better than $0.5 \log \mathrm{MAR}$ in $71 \%$. Variable outcomes between different age groups studied may have been due to phacoemulsification cataract surgery being more difficult in very elderly patients.
Westcott ${ }^{2}$ et al analysed 880 consecutive cataract surgery patients, of whom 263 were over the age of 80 years. The study concluded that not only did the older patients have significantly more surgical complications and less favourable visual outcomes than younger age groups, but also even in the subgroup without coexisting ocular morbidity, the results in the very elderly were less favourable (one-fifth as likely to achieve a VA result of $\geqslant 20 / 40$ as younger patients).

Similarly, Schein ${ }^{3}$ et al found that patients over 75 years of age at surgery were more likely to not improve on measures of visual functioning and acuity postoperatively than younger patients. Berler ${ }^{4}$ found that patients older than 88 years of age were significantly more susceptible to intraoperative complications, such as dropped nuclei and vitreous loss, than younger patients, and that they were less than half as likely as younger patients to achieve a postoperative VA of 20/40 or better.

Monestam $^{5}$ et al found that while best corrected VA increased postoperatively in $94 \%$ of patients over 90 years of age, the increase was significantly lower compared to younger patients (85-89 and $<85$ years old), even after adjusting for other ocular comorbidities.

Improved VA in $79 \%$ of patients over 90 years yielded visual functioning gains in only $47 \%$, thus raising the issue of possible cognition and higher brain-associated visual recognition problems that may affect functional results in the very elderly. ${ }^{6}$

On the other hand, Syam ${ }^{7}$ et al showed improved VA (mean 2.5 Snellen lines) in over $70 \%$ of patients aged 96 years and above, suggesting that ophthalmologists should not be deterred from performing surgery on these patients. Advanced AMD patients (11\%) were the only ones to show visual deterioration, although $62 \%$ of the patients had AMD of varying degree.

In this study, we found that $68 \%$ of patients had improved VA after surgery, $16 \%$ were unchanged, and $16 \%$ had a decrease in VA. As previously mentioned, owing to the structure of our system, patients with operative complications or those needing further treatment of other ocular comorbidities, such as glaucoma and AMD, were more likely to be followed up for longer periods.

VA results after 1 week, especially in this age group, can be misleading, as these patients often present with harder and denser cataracts, loose zonules, and lower endothelial cell counts. This entails significantly longer recovery time after surgery than younger patients. In addition, early examination of uncorrected VA (after a week), even by pinhole examination, is often worse than later corrected VA. Also, we cannot be sure that the final reported VA measurements were truly the best-corrected VA achieved. The better availability of data of the more complicated patients, for longer follow-up, adversely 
Table 2 Changes in visual performance in reference to specific ocular comorbidity

\begin{tabular}{llccc}
\hline & $\begin{array}{c}\text { Comorbidity } \\
\text { Change in VA }\end{array}$ & $\begin{array}{c}\text { No comorbidity and } \\
\text { glaucoma (\%) }\end{array}$ & AMD only (\%) & $\begin{array}{c}\text { P-value } \\
\left(\text { Pearson } \chi^{2}\right)\end{array}$ \\
\hline Preoperative $\rightarrow$ day 7 & Improvement & $21 / 29(72.4)$ & $13 / 22(59.1)$ & $7 / 22(31.8)$ \\
& No change & $1 / 29(3.4)$ & $2 / 22(9.1)$ & 0.015 \\
Preoperative $\rightarrow$ final & Worsening & $7 / 29(24.1)$ & $10 / 16(62.5)$ & 0.134 \\
& Improvement & $20 / 25(80)$ & $4 / 16(25.0)$ & $2 / 16(12.5)$ \\
\hline
\end{tabular}

Merging glaucoma and no comorbidity groups became possible as VA distribution reveal similar values (see Table 3). Statistically significant difference was found between the groups at day 7 .

Table 3 VA parameters categorized by comorbidity groups: AMD patients show less improvement in VA than glaucoma and no comorbidity patients

\begin{tabular}{|c|c|c|c|c|}
\hline Comorbidity (N) & $\begin{array}{c}\text { Preoperative VA } \\
\text { (Snellen equivalent) }\end{array}$ & $\begin{array}{l}\text { Postoperative VA } \\
\text { (Snellen equivalent) }\end{array}$ & $\begin{array}{l}\text { Change in } \\
\text { logMAR }\end{array}$ & P-value \\
\hline \multicolumn{5}{|l|}{ No comorbidity (22) } \\
\hline Mean $( \pm S D)$ & $1.42(\sim 20 / 525) \pm 1.09$ & $0.62(\sim 20 / 82) \pm 0.53$ & -0.80 & \\
\hline Median & $1.0(20 / 200)$ & $0.54(20 / 70)$ & & \\
\hline AMD only (22) & & & & 0.15 \\
\hline Mean $( \pm S D)$ & $1.32(\sim 20 / 415) \pm 0.88$ & $0.83(\sim 20 / 135) \pm 0.64$ & -0.49 & \\
\hline Median & $1.0(20 / 200)$ & $0.70(20 / 100)$ & & \\
\hline Glaucoma only (11) & & & & 0.86 \\
\hline Mean $( \pm S D)$ & $1.35(\sim 20 / 445) \pm 1.01$ & $0.49(\sim 20 / 62) \pm 0.25$ & -0.86 & \\
\hline Median & $1.0(20 / 200)$ & $0.54(20 / 70)$ & & \\
\hline \multicolumn{5}{|l|}{ Total (76) } \\
\hline Mean $( \pm S D)$ & $1.36(\sim 20 / 460) \pm 0.96$ & $0.73(\sim 20 / 108) \pm 0.58$ & -0.63 & \\
\hline Median & $1.0(20 / 200)$ & $0.54(20 / 70)$ & & \\
\hline
\end{tabular}

No statistical significance in VA improvement (change) was found between no comorbidity patients group and AMD or glaucoma patients groups.

affected our final VA results. Data of final VA from the community health care (75\% improvement) support this assumption. Therefore, we speculate that the final overall VA and function of the entire study group are even better than reflected in our results. The natural course of VA in the very elderly should be considered, as visual function is expected to deteriorate with advanced age, even without surgery.

Patients with coexisting AMD showed less improvement in VA (although statistically significant only on day 7) compared to patients with either no ocular disease or with glaucoma at 1 week and at final followup. Yet, over $60 \%$ of AMD patients still showed VA improvement, and mean VA improvement in AMD patients was impressive-0.49 logMAR (from 20/415 to 20/135). Furthermore, visual improvement should also be referred by parameters of quality of vision, colour and contrast discrimination, and the total amount of light entering the eye as well, which can be especially relevant for AMD patients. These patients often report an improvement in the quality of vision and life despite an unimpressive VA improvement. ${ }^{8}$

With regard to operative parameters and intraoperative complications, although this study does not provide a direct randomized prospective comparison with younger patients, we observed lower phacoemulsification $(87.8 \%)$ and posterior chamber IOL implantation $(91.5 \%)$ rates compared to our known overall rates. This indicates several different perioperative surgical considerations (eg, hard and dense cataract, loose zonules, difficult hearing problems, inability to keep still, and cooperate, and so on). Zonulolysis and posterior capsular tears, which occurred in some $12 \%$ of surgeries, were more common than we see in our general surgical population, thus emphasizing weakening of the lens capsule and zonular apparatus with age as possible risk factors in these surgeries. Although this does not justify performing surgery in patients nearing this age group without visually significant cataracts, it may be prudent to operate on 
significant cataracts within a reasonable time rather than postponing surgery to a more advanced age.

In conclusion, our study of 82 consecutive cataract surgeries in patients over 90 years of age shows that, overall, almost $68 \%$ of patients will have a postoperative improvement in their VA, and that if patients with AMD are excluded, this figure rises to over $80 \%$. Special precautions to prevent zonulolysis or posterior capsular tear should be taken, as these were the complications found relatively commonly, whereas other postsurgical complications were not found to be different from our general patient population.

Cataract surgery in these very elderly patients is often rewarding. Improvement in postural stability ${ }^{8,9}$ and reduction of fall-related injuries and risk of fractures ${ }^{10}$ are among the advantages achieved following this operation, and should be considered in the decision to perform cataract surgery in the very elderly.

Justification of surgery may be looked at from a different perspective than for a working adult patient, as preservation and even minor VA improvement can be very valuable for the elderly patient's quality of life. However, ocular and systemic comorbidities, and AMD in particular, must be taken into account when discussing prognosis and expectations with the patient preoperatively.

\section{References}

1 Lundstrom M, Stenevi U, Thorburn W. Cataract surgery in the very elderly. J Cataract Refract Surg 2000; 26: 408-414.

2 Westcott MC, Tuft SJ, Minassian DC. Effect of age on visual outcome following cataract extraction. Br J Ophthalmol 2000; 84: 1380-1382.

3 Schein OD, Steinberg EP, Cassard SD, Tielsch JM, Javitt JC, Sommer A. Predictors of outcome in patients who underwent cataract surgery. Ophthalmology 1995; 102: 817-823.

4 Berler DK. Intraoperative complications during cataract surgery in the very old. Trans Am Ophthalmol Soc 2000; 98: 127-130.

5 Monestam E, Wachmeister L. Impact of cataract surgery on the visual ability of the very old. Am J Ophthalmol 2004; 137: 145-155.

6 Bernth-Petersen P, Ehlers N. Cataract extraction in the 'nineties'. Acta Ophthalmol (Copenh) 1983; 61: 392-396.

7 Syam PP, Eleftheriadis H, Casswell AG, Brittain GP, McLeod BK, Liu CS. Clinical outcome following cataract surgery in very elderly patients. Eye 2004; 18: 59-62.

8 Lundstrom M, Brege KG, Floren I, Lundh B, Stenevi U, Thorburn W. Cataract surgery and quality of life in patients with age related macular degeneration. Br J Ophthalmol 2002; 86: 1330-1335.

9 Schwartz S, Segal O, Barkana Y, Schwesig R, Avni I, Morad $\mathrm{Y}$. The effect of cataract surgery on postural control. Invest Ophthalmol Vis Sci 2005; 46: 920-924.

10 Harwood RH, Foss AJ, Osborn F, Gregson RM, Zaman A, Masud T. Falls and health status in elderly women following first eye cataract surgery: a randomized controlled trial. BR J Ophthalmol 2005; 89: 53-59. 